\title{
Aligned electrospun nanofibers specify the direction of dorsal root ganglia neurite growth
}

\author{
Joseph M. Corey, ${ }_{1}^{1}$ David Y. Lin, ${ }^{2}$ Katherine B. Mycek, ${ }^{1}$ Qiaoran Chen, ${ }^{1}$ Stanley Samuel, ${ }^{3}$ \\ Eva L. Feldman, ${ }^{1}$ David C. Martin ${ }^{2,3,4}$ \\ ${ }^{1}$ Department of Neurology, University of Michigan, Ann Arbor, Michigan 48109 \\ ${ }^{2}$ Macromolecular Science and Engineering Center, University of Michigan, Ann Arbor, Michigan 48109 \\ ${ }^{3}$ Department of Biomedical Engineering, University of Michigan, Ann Arbor, Michigan 48109 \\ ${ }^{4}$ Departments of Materials Science and Engineering; University of Michigan, Ann Arbor, Michigan 48109
}

Received 2 November 2006; revised 29 November 2006; accepted 4 December 2006

Published online 16 May 2007 in Wiley InterScience (www.interscience.wiley.com). DOI: 10.1002/jbm.a.31285

\begin{abstract}
Nerve injury, a significant cause of disability, may be treated more effectively using nerve guidance channels containing longitudinally aligned fibers. Aligned, electrospun nanofibers direct the neurite growth of immortalized neural stem cells, demonstrating potential for directing regenerating neurites. However, no study of neurite guidance on these fibers has yet been performed with primary neurons. Here, we examined neurites from dorsal root ganglia explants on electrospun poly-L-lactate nanofibers of high, intermediate, and random alignment. On aligned fibers, neurites grew radially outward from the ganglia and turned to follow the fibers upon contact. Neurite guidance was robust, with neurites never leaving the fibers to grow on the surrounding cover slip. To compare the alignment of neurites to that of the nanofiber
\end{abstract}

substrates, Fourier methods were used to quantify the alignment. Neurite alignment, however striking, was inferior to fiber alignment on all but the randomly aligned fibers. Neurites on highly aligned substrates were 20 and $16 \%$ longer than neurites on random and intermediate fibers, respectively. Schwann cells on fibers assumed a very narrow morphology compared to those on the surrounding coverslip. The robust neurite guidance demonstrated here is a significant step toward the use of aligned, electrospun nanofibers for nerve regeneration. (C) 2007 Wiley Periodicals, Inc. J Biomed Mater Res 83A: 636-645, 2007

Key words: regeneration; dorsal root ganglia; polylactate; axon; orientation

\section{INTRODUCTION}

Peripheral nerve injury is a significant cause of morbidity. Between 50,000 and 200,000 operations take place each year in the US to repair nerve injuries. ${ }^{1,2}$ Unfortunately, severely injured nerves never reach complete functional recovery. ${ }^{3}$ When gaps between the cut ends of a nerve are greater than $2 \mathrm{~cm}$, a suitable scaffold is required. Nerve autografts produce the best results but often leave patients with significant disability even in optimal conditions. Frequently insufficient in number and size, auto-

Correspondence to: J.M. Corey; e-mail: coreyj@umich.edu

Contract grant sponsor: NIH; contract grant numbers: T32 NSO7222, 1 K08 EB03996, NS36778, NS38849, NINDSN01-NS-1-2338, NSF DMR-0084304

Contract grant sponsor: JDRF Center for the Study of Complications in Diabetes and the Program for Neurology Research and Discovery

(C) 2007 Wiley Periodicals, Inc. grafts produce donor site morbidity. Synthetic nerve guidance channels containing growth factors and extracellular matrix proteins appear to be a promising substitute, but are still limited in the quality of regeneration produced.

Nerve regeneration may be improved by adding fibers that span the length of a guidance channel. ${ }^{4}$ Several studies have demonstrated a powerful ability of fibers to direct neurite outgrowth both in vitro ${ }^{2,5,6}$ and across lesions in peripheral nerve ${ }^{7}$ and spinal cord in vivo. ${ }^{8,9}$ Most of these studies have used fibers greater than $100 \mu \mathrm{m}$ in diameter. ${ }^{2,5-7}$ However, patterned surface chemistry and topography with feature sizes on the cell-length scale (from hundreds of nanometers to tens of microns) affect cell and neurite behavior, including migration, ${ }^{10-15}$ suggesting that fibers in this size range may facilitate neurite elongation. A recent study has shown that fibers with diameters at the size of a cell or smaller, 5 or $30 \mu \mathrm{m}$, produce faster neurite outgrowth than fibers $100 \mu \mathrm{m}$ in diameter or larger. ${ }^{2}$ The ability of yet smaller fibers to guide neurites is just beginning to be explored. 
Electrospinning is a well-established method of producing fibers ranging in diameter from hundreds of nanometers to several microns. Conventional methods of electrospinning use a stationary planar target resulting in mats of randomly oriented fibers, but Yarin and coworkers have recently aligned these fibers by electrospinning on a rapidly rotating wheel. ${ }^{16}$ Numerous studies have shown that a variety of cell types orient parallel to these aligned nanofibers, including fibroblasts, ${ }^{17}$ endothelial cells (19424, 19426, 17180), smooth muscle cells, ${ }^{18}$ osteoblasts, and chondrocytes. ${ }^{19}$ Aligned electrospun fibers also orient the neurites of dissociated cells from the C17.2 neural stem cell line derived from the cerebellum of the mouse. ${ }^{20}$ While this study represents an important first step in investigating the usefulness of aligned nanofibers in a guidance channel, further investigations are needed to demonstrate their suitability in promoting peripheral nerve regeneration. To know that these fibers have such potential, it must be demonstrated that they orient regenerating neurites from the peripheral nervous system (PNS) and that they do so for many neurites simultaneously. Additional questions remain: Do aligned nanofibers change the direction of growth of elongating PNS neurites and continue to direct them toward desired targets? How precisely do neurites follow the alignment of the fibers? Do aligned nanofibers promote faster neurite outgrowth than randomly oriented nanofibers?

To answer these questions, we have electrospun poly-L-lactate (PLLA) fibers of varying alignment and investigated their ability to guide neurites from primary rat dorsal root ganglia (DRG) explants. Neurites that proceed from DRG turn to follow highly aligned fibers and grow along them. Highly aligned fibers increase the speed of neurite outgrowth compared to fibers of intermediate and random alignment. DRG morphology is also affected by fiber alignment, with ganglia on highly aligned fibers becoming elliptical in shape after 3 days, while those grown on randomly aligned fibers remain circular. Analysis of neurite alignment as a function of fiber alignment using Fourier methods demonstrates that neurite alignment approaches, but does not equal, fiber alignment.

\section{MATERIALS AND METHODS}

\section{Electrospinning}

Poly-L-lactide (PLA) with an inherent viscosity of $0.55-$ $0.75 \mathrm{dl} / \mathrm{g}$ was obtained from Birmingham Polymers (Birmingham, AL) and dissolved in chloroform to a concentration of $\sim 3$ wt \%. The polymer solution was delivered by a syringe pump KDS 100 (KD Scientific, New
Hope, PA) with a plastic needle and metal tip, to which an electrode is attached (spinnerette). A voltage of 6-9 kV was applied by a high voltage DC power supply (Hipotronics, Brewster, NY). The target wheel, constructed at the University of Michigan, is $10^{\prime \prime}$ in diameter and has a beveled edge $0.0625^{\prime \prime}$ wide. The wheel was grounded to attract the charged polymer. A motor (Caframo, Wiarton, ON) allows varying the rotation to effect fiber alignment. A $7 \mathrm{~cm}$ distance between the spinnerette and target wheel was used. The wheel was rotated at $250 \mathrm{rpm}$ to produce fibers of high alignment, $110 \mathrm{rpm}$ for intermediate alignment, and $30 \mathrm{rpm}$ for low alignment. Fibers measured $524 \pm 305 \mathrm{~nm}$ (mean \pm std. dev.) in diameter, which ranged from 150 to $1540 \mathrm{~nm}$.

\section{Scanning electron microscopy}

Polymer fibers were first coated with $\sim 100 \AA$ of gold/ palladium by sputtering (Technics Hummer VI). SEM was conducted using a Hitachi S-800, operating in high vacuum at $2 \mathrm{kV}$.

\section{Preparation of fibers for cell culture}

Most fibers were spun directly on to the target wheel and removed and wound on a wooden spool. Fibers were then gently stretched on to $22 \times 22 \mathrm{~mm}^{2}$ glass cover slips and taped taut using small sections of disposable bandage. Some fibers were spun directly onto glass cover slips. For dorsal root ganglia (DRG) experiments, a small volume of collagen (Sigma, St. Louis, MO) was applied to taped fiber bundles and underlying cover slips $(\sim 100 \mu \mathrm{g} / \mathrm{mL}$ in $0.1 \mathrm{M}$ acetic acid) for $30 \mathrm{~min}$ and left to dry.

\section{Culture of primary neurons}

Primary culture was adapted from methods developed in our laboratory. ${ }^{21,22}$ Dorsal root ganglia (DRG) were plucked from the spinal cords of embryonic-day 15 Sprague-Dawley rat embryos and placed directly on the substrate in a minimal amount of media. The culture medium consisted of Neurobasal (Invitrogen) with B27 supplement (Gibco BRL) with the following additives: $30 \mathrm{nM}$ selenium, $10 \mathrm{n} M$ hydrocortisone, $10 \mathrm{n} M \beta$-estradiol, and $10 \mathrm{mg} / \mathrm{L}$ apo-transferrin, and $2 \mu \mathrm{M}$ L-glutamine. A small amount of calf serum (up to $5 \%$ ) was added to promote better explant adhesion and neurite outgrowth. 5-Fluoro2'-deoxyuridine (FUDR), a mitosis inhibitor, was added in some of the experiments to prevent Schwann cell overgrowth.

\section{Neuroblastoma cell culture}

SH-EP cells between passages of 15 and 50 and SHSY5Y cells between passages of 30 and 70 were maintained in Dulbecco's Modified Eagle's Media (DMEM) with 10\% calf serum. Cells were plated at a density of 50 cells $/ \mathrm{mm}^{2}$. 


\section{Immunocytochemistry}

Following culture, cells were fixed in $4 \%$ paraformaldehyde for 15-30 min and stored in $0.1 \mathrm{M}$ sodium phosphate buffer. Samples were soaked in $1 \%$ goat serum and $2 \%$ nonfat dry milk (to block nonspecific binding) and with $0.05 \%$ Triton-X-100 to permeabilize the cells for $15 \mathrm{~min}$. Rabbit antineurofilament (Chemicon, Temecula, CA) was used to label neurons and S-100 (Chemicon), a glial protein, was used to label Schwann cells. In neuroblastoma cells, Oregon-green conjugated phalloidin was used to label actin cytoskeleton. In some experiments, propidium iodide was used to label nuclei. A Nikon Diaphot/FRET system was used for imaging of most samples. For some samples, an Olympus FV500 confocal microscope was used.

\section{Quantification of alignment using Fourier image analysis}

SEM and neurite images were analyzed to quantify the extent of alignment. Electron microscopic images of fibers and dark field images of neurites were taken digitally and opened using ImageJ (http://rsb.info.nih.gov/ij/). Square regions 256 on SEM images of fibers and 512 pixels on fluorescent images of DRG were selected and processed for FFT. FFT images were colorized for publication using a lookup table in ImageJ. A MATLAB script (The MathWorks, Natick, MA) was written to perform the analysis. From the origin of the FFT image, intensities along radii at each angle (at $1^{\circ}$ from the positive $x$-axis to $360^{\circ}$ ) were averaged. The average was calculated in a region ( $\rho$ ) between user specified low and high radius values. For SEM images of fibers, $\rho$ was chosen between 25 and 50 pixels in the FFT, corresponding to a range of 3.17-6.33 $\mu \mathrm{m}$ in the SEM image. Some DRG neurite images were taken with a $10 \times$ objective, however, because waviness of some of the fiber bundles produced large areas of the substrate above or below the focal plane, a smaller region was sometimes captured by changing the objective to $20 \times$. For fluorescence images of neurites, $\rho$ was chosen between 50 and 75 pixels in the FFT, corresponding to a range of 910$455 \mathrm{~nm}$ in DRG neurite images taken with a $10 \times$ objective and a range of $455-228 \mathrm{~nm}$ in the images taken with a $20 \times$ objective.

Average intensities and corresponding angles were stored in an Excel spreadsheet. Average intensity was plotted against angle, generating two intensity peaks. For all data, an 11-point smoother was used on average intensity data to make the graph easier to interpret. The difference between angles on each side of the peak corresponding to one-half the peak height, full width-half max (FWHM) were calculated by averaging minimum and maximum intensities and locating the corresponding angle using the cursor, which caused the angle and intensity to be displayed. FWHM varied by $1-2^{\circ}$, unless $\rho$ was made large enough to nearly envelop the entire FFT, in which case it frequently improved by $3-4^{\circ}$. Statistics were calculated using Prism 3 software (www.graphpad.com). One-way analysis of variance was used to minimize chance of type-I error when comparing alignment among groups, and a
Tukey's multiple comparison test was used to compare alignment between two groups.

\section{Measurement of neurite length and ganglia}

DRG explants were grown for 3 days on fibers of high, intermediate, and random alignment. After immunostaining, photographs of whole explants including neurites were taken on a Leica MZFL III stereo dissecting fluorescent microscope. The number of explants measured was 11 on fibers of intermediate alignment and 12 each on both highly and randomly aligned fibers. Using Metamorph software, the perimeter around each ganglion was marked and measured. Ten distinct neurites were chosen to measure from each explant. Neurites selected were solitary and clearly delineated to avoid confusion with other neurites; also, neurites were distributed approximately equally around the perimeter of the ganglion. Each neurite was measured from its origin at the marked perimeter of the ganglion to its end by tracing along its entire length to account for changes in direction along its course.

To assess the shape of the ganglia, the length and width of each ganglion was measured. Measurements were made from one end of the perimeter of the ganglion through its center to the other end. The length was measured parallel to the fiber bundle and the width was measured perpendicular to the fiber bundle. Aspect ratios, defined as length divided by width, were calculated for each ganglion.

\section{RESULTS}

\section{Electrospun PLLA fibers}

We first electrospun fibers into an aligned morphology. We selected poly-L-lactate (PLLA) as the material for our neuron scaffolds because of its known biocompatibility and degradability. PLLA dissolved in chloroform was electrospun on to a rotating stainless steel target wheel, $25 \mathrm{~cm}$ in diameter, with an edge beveled to $1.6 \mathrm{~mm}$. Scanning electron microscopy (SEM) shows that PLLA fibers are highly aligned when electrospun at 1000 rotations per minute [Fig. 1(A)].

\section{Dorsal root ganglia explants on aligned PLLA fibers}

We hypothesized that neurites from cultured DRG explants would extend in random directions, come into contact with the fibers, and then turn to follow them [Fig. 1(B)]. After attachment of the explants to the fiber bundle, neurites emanate from the explant radially [Fig. 1(C,D)]. Upon contacting the fibers [Fig. 1(C)], neurites change direction and grow parallel to them [Fig. 1(D)]. Some DRG explants adhere to the cover slip adjacent to the fiber bundle. On these 

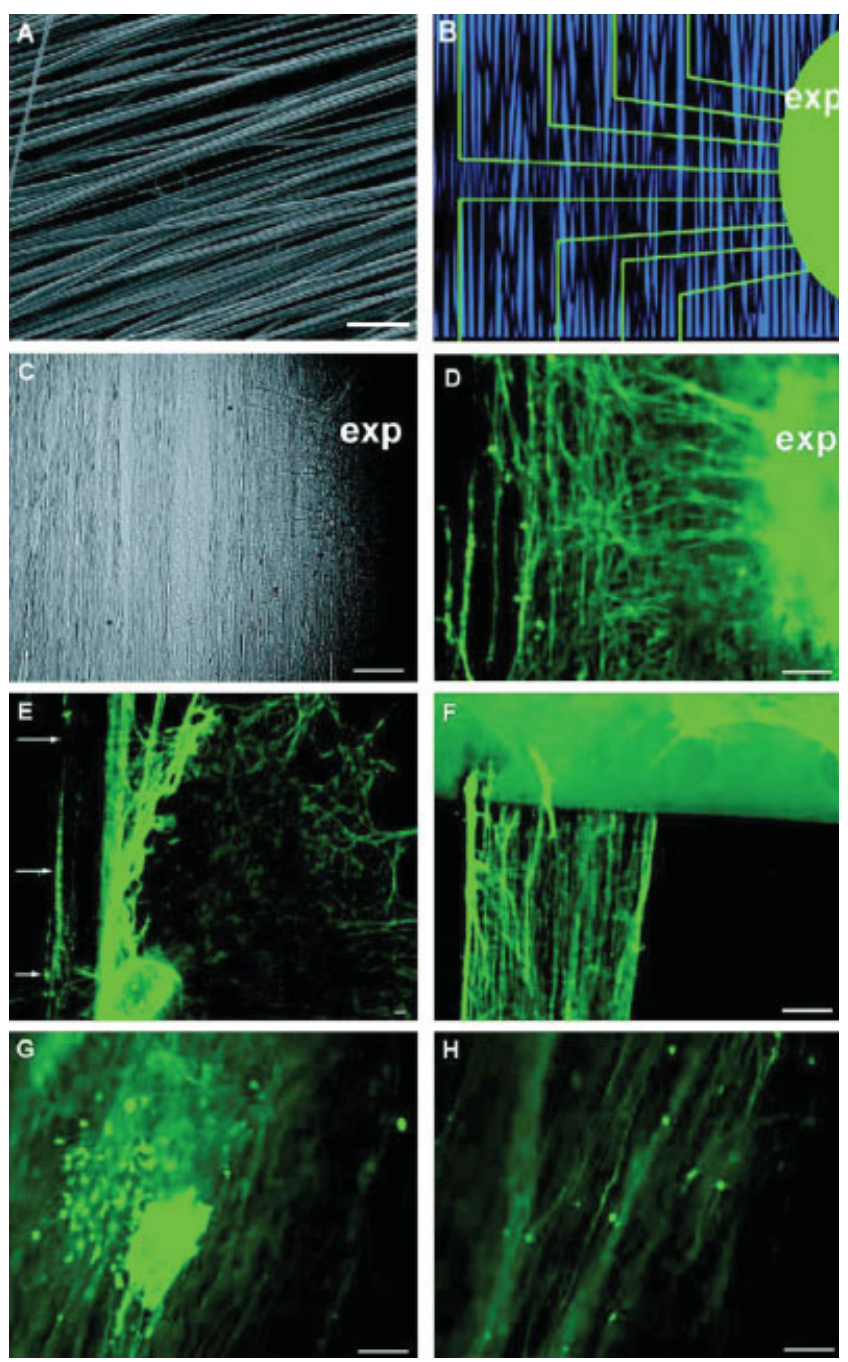

Figure 1. Aligned PLLA nanofibers guide regenerating peripheral nervous tissue. DRG were plucked from the spinal cords of embryonic 15-day rats and placed on collagen-coated fiber bundles. A: SEM photo of aligned PLLA fibers used in these experiments. B: Hypothesized behavior of neurite extension from DRG explant. C: DRG explant (exp) on aligned PLLA fibers as seen with transmitted light. D: Neurofilament-stained neurites in the same area as (C), extend from the large explant (exp) on the right and continue outward until they contact collagen-coated PLLA fibers, then turn to follow them vertically. Note that all neurites on the left side of the photograph travel vertically. E: A DRG explant on glass adjacent to a fiber extends neurites radially on the glass (right side of the photo), while neurites contacting the fiber bundle (left side of the photo) follow the fibers. Arrows point to left border of the fiber bundle. F: Neurites migrating up to the edge of the anchoring tape holding down the fiber bundle do not continue to grow on it nor leave the fibers for the glass, despite their being coated with collagen. G: By 12 days, cell bodies of neurons in the DRG explants break up, but neurites remain on fibers, even over $10 \mathrm{~mm}$ away. $\mathrm{H}$ : The appearance of cell bodies can be found at least $100 \mu \mathrm{m}$ away from the explant in $G$, demonstrating migration along the fibers. Scale bar for $\mathrm{A}$ is $20 \mu \mathrm{m}$ and for $\mathrm{C}-\mathrm{H}$ is $100 \mu \mathrm{m}$. [Color figure can be viewed in the online issue, which is available at www.interscience.wiley.com.] explants, neurites adjacent to the bundle contact the fibers and are guided by them, while neurites on other sides proceed outward, forming a dense net of unguided neurites [Fig. 1(E)]. Neurites on aligned fibers extend linearly and appear to extend farther than those on glass, which grow to form a characteristic web-like morphology. Once contacting the fibers, neurites continue to follow them, never leaving to follow collagen-coated glass or the anchoring tape, even after culture periods longer than 12 days [Fig. 1(F)]. Cell bodies in the DRG grown on fibers for 10 days gradually dissociate and distance themselves from the explants, but their neurites continue to remain localized in the fiber bundle [Fig. $1(\mathrm{G}, \mathrm{H})]$.

\section{Neurite alignment as a function of fiber alignment}

To investigate the dependence of neurite alignment on fiber alignment, DRG were cultured on bundles of fibers electrospun with differing orientations. Compared to the aligned neurites observed on the aligned fibers, fibers of intermediate and then random alignments produced successively inferior neurite alignment [Fig. 2(A-C,G-I)].

To quantify this observation, analyses of both fiber and neurite alignment were performed in the frequency domain by first performing a FFT of the spatial image [Fig. 2(D-F,J-L)] and then determining the full-width-half max (FWHM) of the intensities in the FFT image [Fig. 2(M-O)]. Highly aligned fibers (FWHM of $23^{\circ} \pm 6^{\circ}$ ) produced highly aligned neurites (FWHM of $38^{\circ} \pm 7^{\circ}$ ) (mean \pm S.D.). Fibers of intermediate (FWHM of $46^{\circ} \pm 15^{\circ}$ ) and random alignment (FWHM of $78^{\circ} \pm 22^{\circ}$ ) produced correspondingly poorer neurite alignment (FWHM of $69^{\circ}$ $\pm 18^{\circ}$ and $84^{\circ} \pm 25^{\circ}$, respectively). Fiber alignment has a profound effect on neurite alignment among the three groups $(p<0.0001)$. Highly aligned fibers were superior to those with intermediate and random alignment $(p<0.001)$ and fibers of intermediate alignment were superior to those with random alignment $(p<0.05)$. In all three cases, neurite alignment was less than fiber alignment.

\section{Effect of fiber alignment on neurite length and ganglion shape}

We investigated whether fiber alignment increases the speed of neurite outgrowth by using Metamorph software to measure ten individual neurites proceeding from each ganglion. Neurites on the highly aligned fibers were longer than neurites on lesser aligned fibers, measuring $760 \pm 71 \mu \mathrm{m}(p<0.001)$ (Fig. 3). The lengths of neurites grown on intermediate and random fibers were not statistically different, measuring $656 \pm 52 \mu \mathrm{m}$ and $631 \pm 32 \mu \mathrm{m}$, respec- 

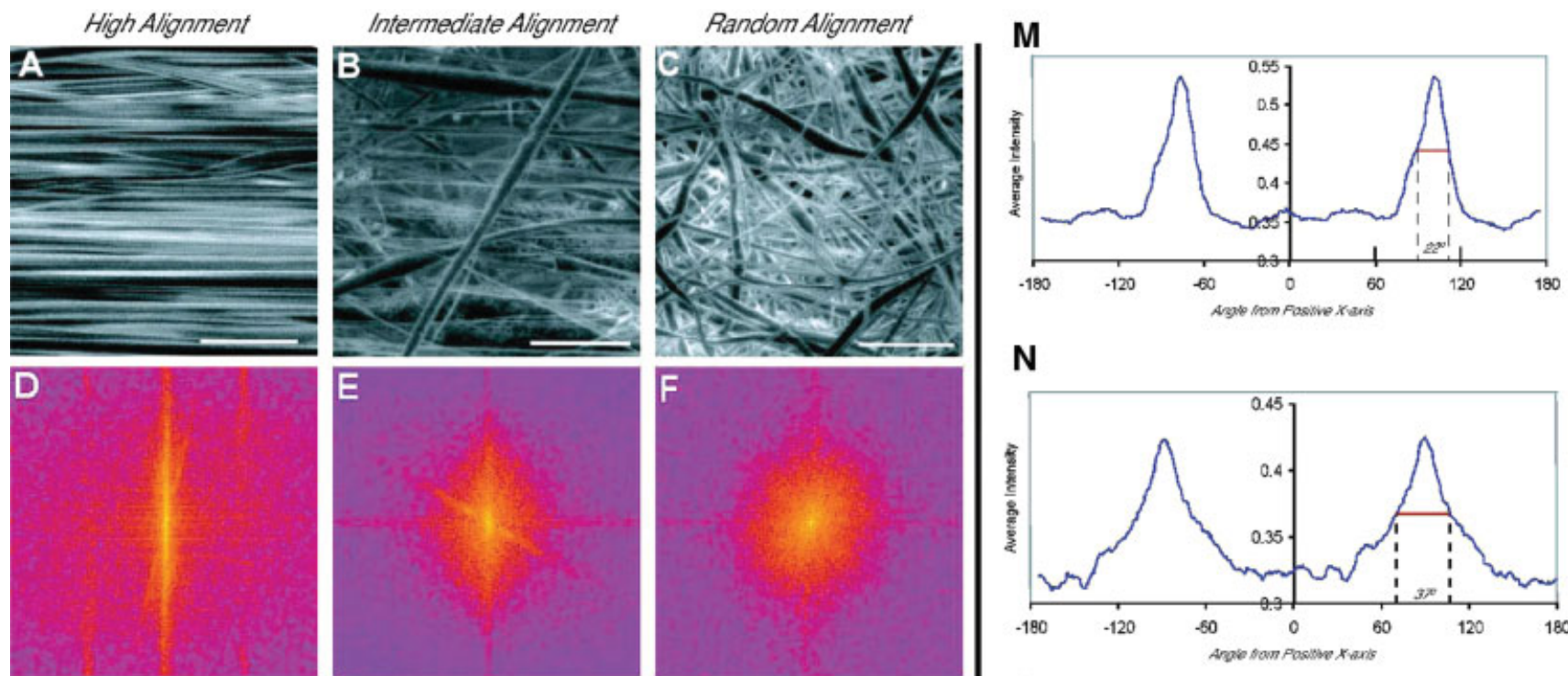

N
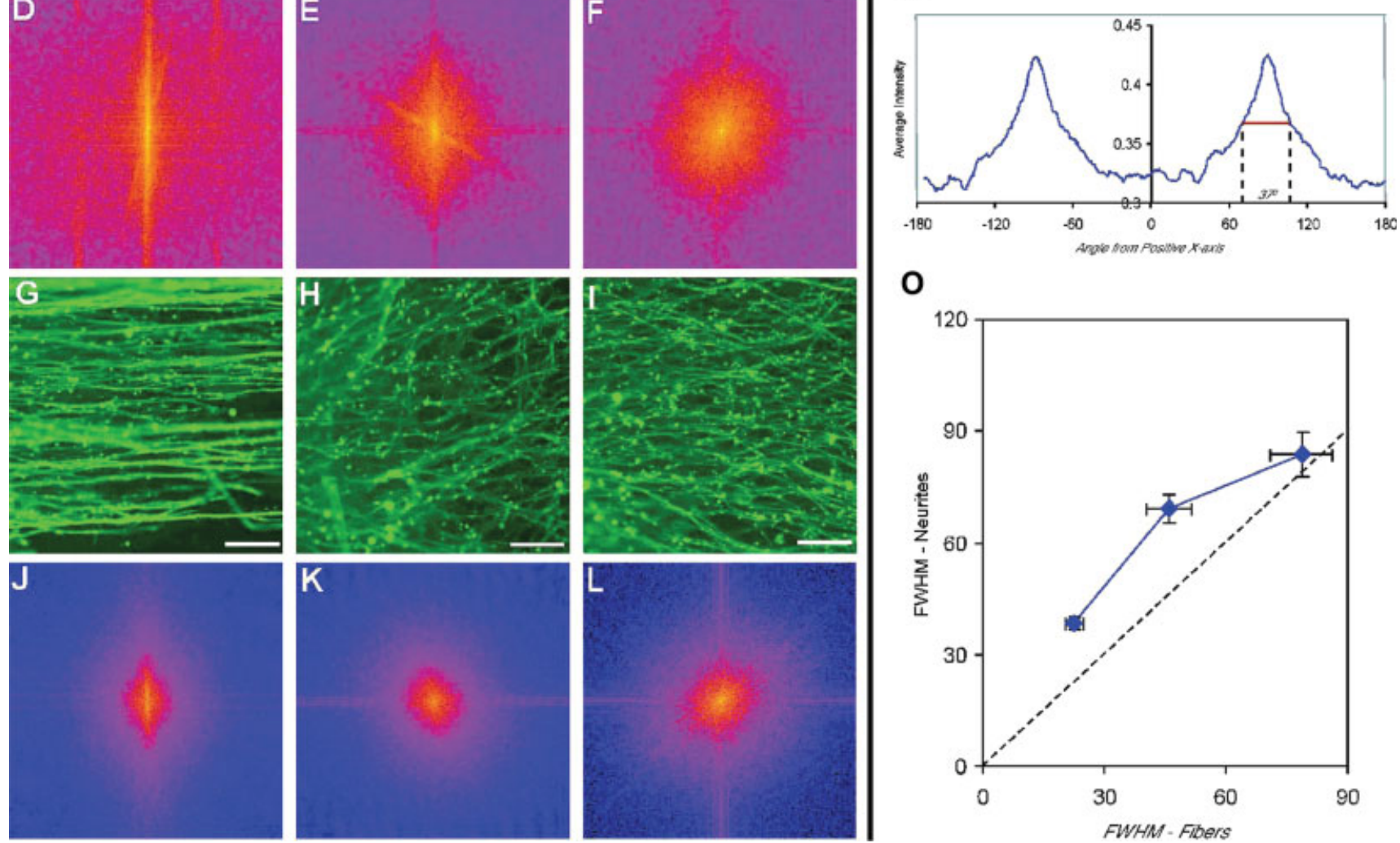

Figure 2. Alignment of neurites is dependent on the alignment of underlying nanofibers. A-C: SEM images of fibers with high (A), intermediate (B), or low (C) alignment. D-F: FFT images from $256 \times 256$ pixel selections from the images in A-C, respectively. Yellow depicts greatest intensity, blue depicts least intensity. Note that narrower areas of higher intensity (yellow-orange) in the FFT images correlate with more oriented fibers. G-I: Neurites from DRG grown for 3 days on fibers of identical alignments as depicted in A-C. J-L: FFT images from $512 \times 512$ pixel selections from the images in G-I. M: Graph of average intensity of D (FFT of A) that lies within a circle between 12 and 25 pixels from the center of the FFT image, as a function of angle from the positive X-axis, displaying a full width-half max (FWHM) of $22^{\circ}$. N: Graph of average intensity of J (FFT of G) that lies within a circle between 50 and 75 pixels from the center of the FFT as a function of angle from the positive X-axis, displaying a FWHM of $37^{\circ}$. O: Plot of orientation (FWHM) of neurites versus fibers for all three levels of fiber orientation. For both high and intermediate alignment, neurites are not as well aligned as the fibers. Data plotted are mean with horizontal and vertical error bars (standard error of measurement). The dashed line indicates equality between FWHM of neurites and fibers. For fibers, $N=8$ samples for all alignments. For neurites, $N=$ 15 for highly aligned fibers, $N=23$ for intermediate alignment, and $N=18$ for unaligned fibers. Scale bars for A-C are $10 \mu \mathrm{m}$ and G-I are $100 \mu \mathrm{m}$. [Color figure can be viewed in the online issue, which is available at www.interscience. wiley.com.]

tively. Using these measures, highly aligned fibers increased the rate of neurite growth by $20 \%$ compared to randomly aligned fibers and $16 \%$ compared to fibers of intermediate alignment.

Fiber alignment also affected the shape of the ganglia. On initial observation, the ganglia on highly aligned fibers appeared to elongate parallel to the fibers, while simultaneously becoming narrower perpendicular to the fibers. Those on randomly aligned fibers appeared circular in shape (Fig. 3). The length and width of each ganglion were measured. While the perimeters of the ganglia were equal on all three fiber types, the ganglia were elongated in the direction of alignment (Fig. 3). Ganglia on highly aligned 

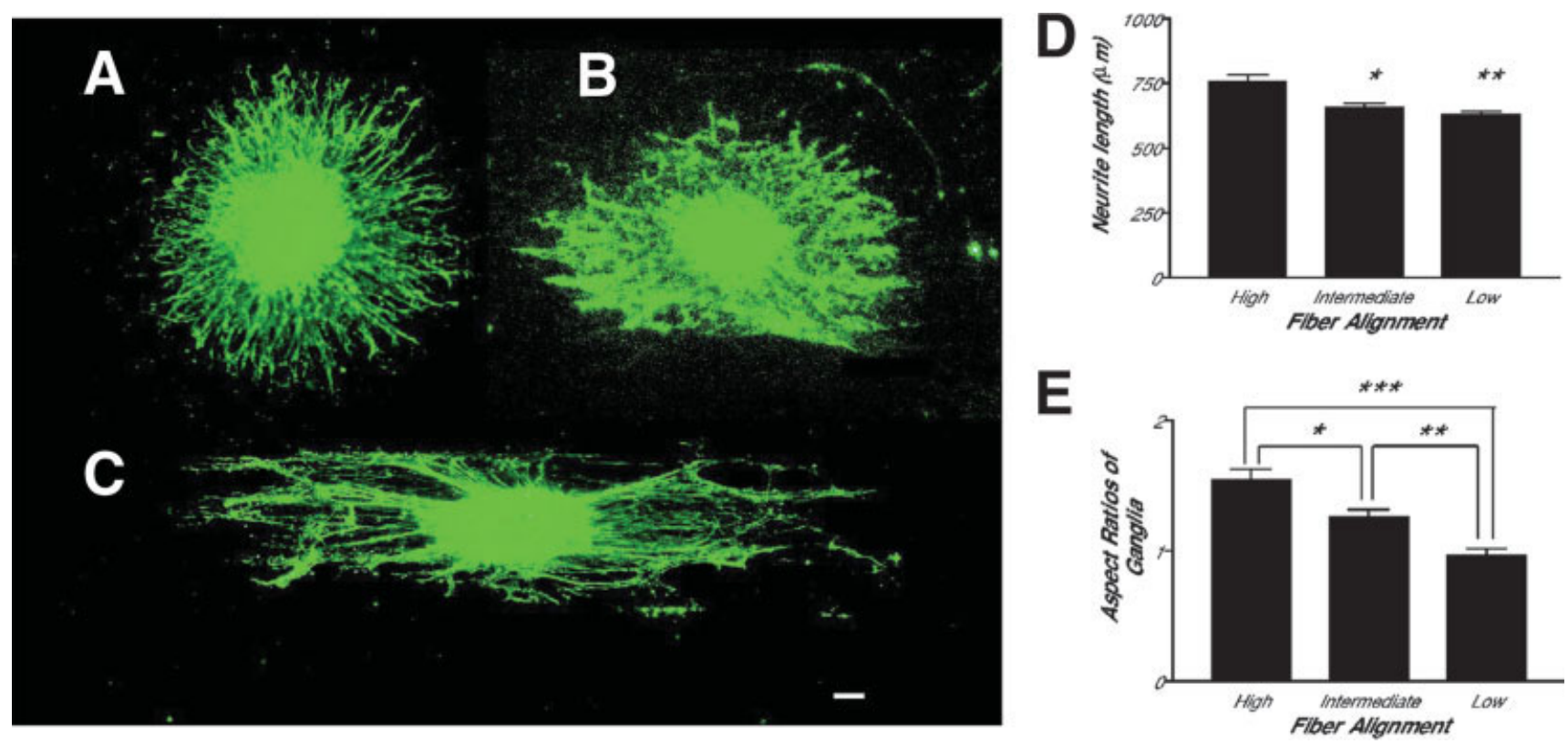

Figure 3. Effects of fiber alignment on neurite length and ganglia shape. DRG grown for 3 days and then stained for neurofilament are shown on randomly aligned fibers (A), intermediate fibers (B), and highly aligned fibers (C). D: Neurites on highly aligned fibers grew farther than those on intermediate and random fibers. The aspect ratio (length:width) of ganglia was greatest on highly aligned fibers, somewhat increased on intermediate fibers, and 1 on random fibers. Scale bar is $100 \mu \mathrm{m} .{ }^{*} p<0.05,{ }^{* *} p<0.01,{ }^{* *} p<0.001$. [Color figure can be viewed in the online issue, which is available at www. interscience.wiley.com.]

fibers were the most elliptical in shape; those on intermediate fibers were elliptical, but less so. Aspect ratios of ganglia cultured on fiber bundles of high, intermediate, and random alignments were calculated as 1.54, 1.26, and 0.966 , respectively $(p<$ 0.0001, ANOVA).

\section{Schwann cells on PLLA fibers}

Some dorsal root ganglia were stained with antibody to s100, a glial marker that is expressed in Schwann cells. Effects of fibers on rat Schwann cell morphology can be clearly seen in Fig. 4. Schwann cells adhering to planar glass exhibit good spreading, while those adhering to fibers elongate and become extremely narrow. Schwann cells have been observed elongating away from the explant and trail slightly behind the advancing neurites (data not shown).

\section{Neuroblastoma cells on PLLA fibers}

Yang et al. used an immortalized neural stem cell line that did not require adhesion-promoting pro-
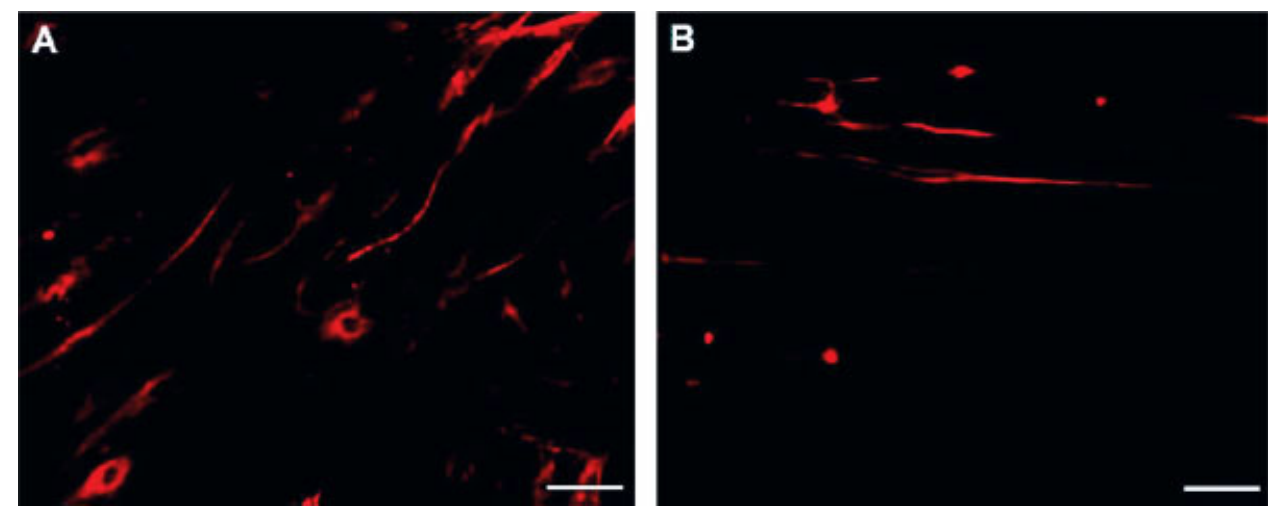

Figure 4. Effects of aligned nanofibers on Schwann cells from DRG explants. A: Schwann cells on collagen-coated glass stained with S-100 antibody and rhodamine exhibit good spreading, while cells on fibers (B) conform to them and are extremely narrow. Scale bars are $50 \mu \mathrm{m}$. [Color figure can be viewed in the online issue, which is available at www. interscience.wiley.com.] 

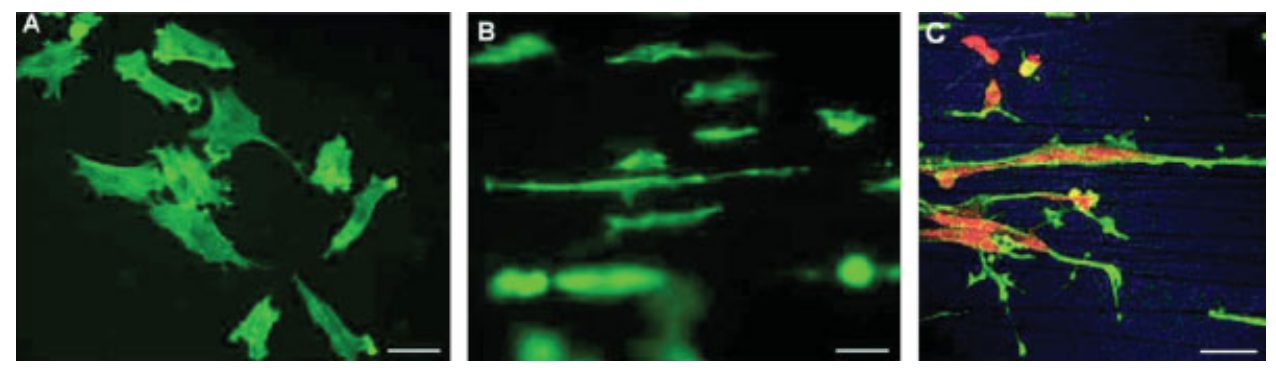

Figure 5. Effects of aligned nanofibers on transformed neuroblasts. A: SH-EP cells plated on the flat cover slip adjacent to PLLA fibers are well spread and oriented randomly. B: Cells adhering to the fiber bundle are longer and narrower. Actin stress fibers run parallel to the long axes of the cells and to the fibers. Note that some cells are out of focus, indicating their localization at various depths of the fiber bundle. C: The cell bodies and neurites of SH-SY5Y cells also orient to follow the sparser fibers spun on glass. Scale bars are $100 \mu \mathrm{m}$. [Color figure can be viewed in the online issue, which is available at www.interscience.wiley.com.]

teins or peptides to attach and grow. ${ }^{20}$ To test aligned fibers for their influence on individual cells that do not require adhesive coatings, we used two well-characterized, human neuroblastoma cell lines, SH-EP and SH-SY5Y. SH-EP cells on planar glass spread in all directions with the long axes of the cells oriented in random directions and stress fibers pointing to focal contacts [Fig. 5(A)]. On aligned PLLA fibers, cells were elongated and narrow, with the long axes of cells and the internal actin stress fibers oriented parallel to the fibers [Fig. 5(B)]. Less dense nanofibers, formed by electrospinning directly on glass cover slips taped to the rotating target wheel, also influence cell orientation. SH-SY5Y cells grown on these low-density aligned fibers oriented their cell bodies and neurites parallel to them [Fig. $5(\mathrm{C})]$.

\section{DISCUSSION}

This is the first demonstration of guided neurite outgrowth from primary nervous tissue on aligned, electrospun nanofibers. Neurites from DRG explants elongate radially from the ganglion, but turn to follow the fibers upon contact and do not leave them to grow on adjacent, smoother surfaces even after a week of growth. Neurite alignment, although striking, was never as pronounced as the alignment of underlying fibers. Additionally, highly aligned fibers facilitated faster neurite outgrowth than fibers of intermediate and random alignment. The shape of the ganglia shape was also affected by fiber alignment, with ganglia elongating along the fibers. This is also the first study where tissue explants, as opposed to dissociated cells, have been cultured on aligned nanofibers.

Structures with longitudinal aligned topography have shown very promising results in supporting neuronal growth across lesions in vivo. ${ }^{23}$ The likely mechanism for the success of these materials is the alignment of glia and continual contact guidance along the length of the scaffold. ${ }^{2}$ Our fibers caused alignment of Schwann cells and provided a topographical guidance cue for neurites. The radial neurite growth from DRG appears to be directed along the fibers upon or soon after neurite contact. This direction growing neurites is demonstrated particularly well on explants that contact both the PLLA fibers on one side and the flat glass substrates underlying them on the other side [Fig. 1(E)]. Neurites never leave the fiber bundle to migrate on the glass. These results are consistent with the principle that neurite growth is superior when neurites encounter anisotropic cues in the microenvironment. ${ }^{1}$ The topographies of nanofibers used in our study likely resemble features that spur neurite migration in vivo, including the extracellular matrix present in nerve autografts. Neurites grow between the fibers in the fiber bundle, as we have observed that they lie at different focal lengths in the bundle (data not shown). It is possible that Schwann cells and growth cones sense this contact guidance cue not only below, but also above and to each side, supplying a stronger cue to spur their migration down the fiber bundle.

On biomaterials, feature sizes on the cell-length scale affect cell behavior. Of fibers studied for their ability to guide neurites, most are greater in diameter than $100 \mu \mathrm{m},{ }^{2,5-7}$ much larger than the diameter of neuronal cell bodies and neurites. Only a few studies have been performed with fibers in the cell length scale or below. ${ }^{2,8,9}$ However, there is substantial evidence from studies on planar substrates using patterned surface chemistry and topography to suggest that features on the cell-length scale affect cell migration and neurite outgrowth. On patterned polylysine grids, Corey et al. ${ }^{10}$ showed hippocampal neurons migrate faster to areas of larger adhesion when traveling on a line $5 \mu \mathrm{m}$ wide than when traveling on a line 10 or $3 \mu \mathrm{m}$ wide. Li et al. ${ }^{12}$ showed 
that endothelial cells are narrower and more likely to migrate on collagen lines that are $15 \mu \mathrm{m}$ wide as opposed to 30 or $60 \mu \mathrm{m}$. Neurites are all effectively guided by lines between 2 and $5 \mu \mathrm{m} \cdot{ }^{10,24,25}$ Using fibers, Wen and Tresco ${ }^{2}$ showed similar results comparing Schwann cell and neurite migration along fibers 5, 30, 100, 200, and $500 \mu \mathrm{m}$ in diameter. Whether precoated with fibronectin or laminin, the narrowest fibers produced the farthest Schwann cell migration and the longest neurite outgrowth along the fiber.

The ability of feature size to affect neurite growth and cell development extends into the nanometer range. In the first study to examine neurite outgrowth on aligned, electrospun fibers, neurites of C17.2 neural stem cells were guided by fibers ranging in diameter from $700 \mathrm{~nm}$ to $3.5 \mu \mathrm{m} .{ }^{20}$ Differentiation of the stem cells occurred faster on the aligned nanofibers than on aligned microfibers. The morphology of these cells was largely bipolar on aligned nanofibers, while those on planar surfaces were frequently multipolar. However, this behavior may be cell type or species specific. As an example, in a study of neurite growth on aligned grooves on planar surfaces, Xenopus neurites grew parallel to grooves $14 \mathrm{~nm}$ deep and $1 \mu \mathrm{m}$ wide, but cultured hippocampal neurons grew perpendicular to them. ${ }^{15}$ However, neurites extending from chick cerebral neurons do not follow grooves $130 \mathrm{~nm}$ wide even when the depth of the grooves is $400 \mathrm{~nm} .^{13}$ These studies, together with our data, demonstrate that neurites are guided by nanometer-scale topographies, but also suggest an underlying limit to the size of features that guide neurites.

Ours is the first study to test aligned nanofibers with primary neurons and is an important step in using such fibers as a tool for nerve regeneration. The C17.2 neural stem cell line used by Yang et al. ${ }^{20}$ is an immortalized line that differs significantly from the primary nervous tissue used in our study. This line is very attractive, having been instrumental in producing regeneration with improvement in functional recovery when implanted on a scaffold in spinal cord injured rats. ${ }^{23}$ This may be due in part to the secretion of trophic factors demonstrated by the powerful promotion of nerve regeneration when implanted into the distal segment of a transected peripheral nerve. ${ }^{26}$ However, recent investigation suggests that the unique properties of this line cannot be generalized to other stem cells or neurons. ${ }^{27}$ C17.2 cells secrete growth factors and cytokines not secreted by other neural stem cells. In addition, they have karyotypic instability. Yang et al. $^{20}$ reported that they adhere to electrospun fibers without prior coating of extracellular matrix proteins or polyamino acids required by primary neurons, demonstrating a profound difference in phenotype. In our initial testing of these fibers, we used cells from two human neuroblastoma lines without prior surface coating and observed that they orient their cell bodies and extend processes parallel to the fibers. This difference in cell-to-substratum adhesion strongly suggests that primary neurons are a more predictive model of neurite outgrowth from peripheral nerve.

In our experiments comparing neurite alignment on PLLA fiber alignment, there appears to be a limit in how closely neurites will follow fibers even when the fibers are well aligned. There are likely several explanations for this. First, neurites likely follow one fiber for a time then follow another intersecting fiber. The packing of fibers in the bundle likely increases as alignment increases, leaving less space between the fibers and giving neurites several fibers from which to choose. As intimated above, neurites may grow in the spaces between fibers. Second, collagen is applied to the fiber bundle as a whole, not to individual fibers. The resulting film may cover the fibers in such a way as to smooth their topography but produce valleys in the collagen film. These may be what effectively guide the neurites instead of the fibers themselves. Third, fibers are not the only guidance cue. Neurites can follow other neurites, and may be more likely to fasciculate with time. Fourth, neurites proceed radially from the DRG cell bodies in the explant, giving them an initial organization in space that the fibers must overcome [Fig. 1(E)].

The increased speed seen in neurite growth on highly aligned fibers is consistent with that seen in C17 cells by Yang et al. ${ }^{20}$ They reported that neurites on aligned nanofibers averaged $100 \mu \mathrm{m}$ and that those on random fibers averaged $80 \mu \mathrm{m}$, an increase of $25 \%$ due to alignment. On our fibers, we saw an increase of $20 \%$ due to alignment. The increased speed on aligned fibers is likely due to alignment. Neurite growth slows when growth cones make choices between two paths or materials. ${ }^{28,29}$ Growth cones may gain either reinforcing or conflicting guidance cues when contacting two or more fibers simultaneously. We speculate that the larger the angle between two adjacent fibers contacted by the same growth cone, the more time the growth cone may take to choose between the fibers. Since random fibers possess greater angles between adjacent fibers, growth cones cultured on them likely have to spend more time to make a choice among them, thus decreasing the time spent elongating the neurite.

A very interesting and unexpected result was the change in the shape of the ganglia as function of the alignment of the fibers on which they were cultured. It has been well established that the cell shape is altered by feature sizes on patterns and topographies. ${ }^{12,30-32}$ Fibroblasts in the fibrous capsule on the exterior of ganglia contact the fiber bundle and likely 
orient parallel to the fibers during culture, perhaps changing the shape of the explant. Another potential reason for this behavior follows from the mechanics of neurite extension which is directly proportional to cytoskeletal tension (Heidemann et al., 1995). ${ }^{33}$ Since the majority of neurites exerts tension parallel to the fiber bundle, the tension would encourage deformation of the ganglion in the direction of the fibers. Neurites grown on randomly oriented fibers pull in all directions, leaving the ganglion in the shape of a circle.

Electrospinning is a straightforward method for creating longitudinally oriented nanofibers with potential for nerve regeneration. Major gains in the science of nerve regeneration are being made using guidance channels that rely on cell-mediated release of nerve growth factor, ${ }^{33}$ slow release of NGF with laminin-containing gels, ${ }^{34}$ or aligned gels of laminin and collagen. ${ }^{35}$ Combining these existing technologies with aligned fibers may provide superior regeneration in the PNS and novel approaches for solving the more difficult problem of regeneration in the spinal cord and brain.

The authors would like to thank Dr. Steven Lentz of the Michigan Diabetes Research and Training Center, and Dr. Kelli Sullivan and John Hayes of The Juvenile Diabetes Research Foundation Center for the Study of Complications in Diabetes, Morphology Image Analysis Core. We also wish to thank Carey Backus and Dr. Gina Leinninger for their assistance in DRG culture. We are also appreciative of the University of Michigan Electron Microbeam Analysis Laboratory (EMAL).

\section{References}

1. Bellamkonda RV. Peripheral nerve regeneration: An opinion on channels, scaffolds and anisotropy. Biomaterials 2006; 27:3515-3518.

2. Wen X, Tresco PA. Effect of filament diameter and extracellular matrix molecule precoating on neurite outgrowth and Schwann cell behavior on multifilament entubulation bridging device in vitro. J Biomed Mater Res A 2006;76:626-637.

3. Frostick SP, Yin Q, Kemp GJ. Schwann cells, neurotrophic factors, and peripheral nerve regeneration. Microsurgery 1998;18:397-405.

4. Hilburger EW, Conte EJ, McGee DW, Tammariello SP. Localization of NADPH oxidase subunits in neonatal sympathetic neurons. Neurosci Lett 2005;377:16-19.

5. Rangappa N, Romero A, Nelson KD, Eberhart RC, Smith GM. Laminin-coated poly(L-lactide) filaments induce robust neurite growth while providing directional orientation. J Biomed Mater Res 2000;51:625-634.

6. Steuer H, Fadale R, Muller E, Muller HW, Planck H, Schlosshauer B. Biohybride nerve guide for regeneration: Degradable polylactide fibers coated with rat Schwann cells. Neurosci Lett 1999;277:165-168.

7. Lundborg G, Dahlin L, Dohi D, Kanje M, Terada N. A new type of "bioartificial" nerve graft for bridging extended defects in nerves. J Hand Surg [Br] 1997;22:299-303.

8. Zhang YH, Kenyon JL, Nicol GD. Phorbol ester-induced inhibition of potassium currents in rat sensory neurons requires voltage-dependent entry of calcium. J Neurophysiol 2001;85: 362-373.
9. Chauhan NB, Figlewicz HM, Khan T. Carbon filaments direct the growth of postlesional plastic axons after spinal cord injury. Int J Dev Neurosci 1999;17:255-264.

10. Corey JM, Wheeler BC, Brewer GJ. Compliance of hippocampal neurons to patterned substrate networks. J Neurosci Res 1991;30:300-307.

11. Corey JM, Wheeler BC, Brewer GJ. Micrometer resolution silane-based patterning of hippocampal neurons: Critical variables in photoresist and laser ablation processes for substrate fabrication. IEEE Trans Biomed Eng 1996;43:944-955.

12. Li S, Bhatia $\mathrm{S}, \mathrm{Hu} \mathrm{YL}$, Shiu YT, Li YS, Usami S, Chien S. Effects of morphological patterning on endothelial cell migration. Biorheology 2001;38:101-108.

13. Clark P, Connolly P, Curtis AS, Dow JA, Wilkinson CD. Cell guidance by ultrafine topography in vitro. J Cell Sci 1991; 99(Pt 1):73-77.

14. Curtis A, Wilkinson C. Topographical control of cells. Biomaterials 1997;18:1573-1583.

15. Rajnicek A, Britland S, McCaig C. Contact guidance of CNS neurites on grooved quartz: Influence of groove dimensions, neuronal age and cell type. J Cell Sci 1997;110(Pt 23):2905-2913.

16. Theron A, Zussman E, Yarin AL. Electrostatic field-assisted alignment of electrospun nanofibres. Nanotechnology 2001; 12:384-390.

17. Lee $\mathrm{CH}$, Shin HJ, Cho IH, Kang YM, Kim IA, Park KD, Shin JW. Nanofiber alignment and direction of mechanical strain affect the ECM production of human ACL fibroblast. Biomaterials 2005;26:1261-1270.

18. Baker SC, Atkin N, Gunning PA, Granville N, Wilson K, Wilson $\mathrm{D}$, Southgate J. Characterisation of electrospun polystyrene scaffolds for three-dimensional in vitro biological studies. Biomaterials 2006;27:3136-3146.

19. Bhattarai N, Edmondson D, Veiseh O, Matsen FA, Zhang M. Electrospun chitosan-based nanofibers and their cellular compatibility. Biomaterials 2005;26:6176-6184.

20. Yang F, Murugan R, Wang S, Ramakrishna S. Electrospinning of nano/microscale poly(L-lactic acid) aligned fibers and their potential in neural tissue engineering. Biomaterials 2005; 26:2603-2610.

21. Russell JW, Windebank AJ, Schenone A, Feldman EL. Insulin-like growth factor-I prevents apoptosis in neurons after nerve growth factor withdrawal. J Neurobiol 1998;36:455-467.

22. Russell JW, Golovoy D, Vincent AM, Mahendru P, Olzmann JA, Mentzer A, Feldman EL. High glucose induced oxidative stress and mitochondrial dysfunction in neurons. FASEB J 2002;16:1738-1748.

23. Teng YD, Lavik EB, Qu X, Park KI, Ourednik J, Zurakowski D, Langer R, Snyder EY. Functional recovery following traumatic spinal cord injury mediated by a unique polymer scaffold seeded with neural stem cells. Proc Natl Acad Sci USA 2002;99:3024-3029.

24. Clark P, Britland S, Connolly P. Growth cone guidance and neuron morphology on micropatterned laminin surfaces. J Cell Sci 1993;105(Pt 1):203-212.

25. Ma W, Liu QY, Jung D, Manos P, Pancrazio JJ, Schaffner AE, Barker JL, Stenger DA. Central neuronal synapse formation on micropatterned surfaces. Brain Res Dev Brain Res 1998;111:231-243.

26. Heine W, Conant K, Griffin JW, Hoke A. Transplanted neural stem cells promote axonal regeneration through chronically denervated peripheral nerves. Exp Neurol 2004;189:231-240.

27. Mi R, Luo Y, Cai J, Limke TL, Rao MS, Hoke A. Immortalized neural stem cells differ from nonimmortalized cortical neurospheres and cerebellar granule cell progenitors. Exp Neurol 2005;194:301-319.

28. Esch T, Lemmon V, Banker G. Local presentation of substrate molecules directs axon specification by cultured hippocampal neurons. J Neurosci 1999;19:6417-6426. 
29. Gomez TM, Letourneau PC. Filopodia initiate choices made by sensory neuron growth cones at laminin/fibronectin borders in vitro. J Neurosci 1994;14:5959-5972.

30. Dike LE, Chen CS, Mrksich M, Tien J, Whitesides GM, Ingber DE. Geometric control of switching between growth, apoptosis, and differentiation during angiogenesis using micropatterned substrates. In Vitro Cell Dev Biol Anim 1999;35: 441-448.

31. Chen CS, Mrksich M, Huang S, Whitesides GM, Ingber DE. Geometric control of cell life and death. Science 1997;276: $1425-1428$.
32. Thompson DM, Buettner HM. Schwann cell response to micropatterned laminin surfaces. Tissue Eng 2001;7:247-265.

33. Heidemann SR, Lamoreux P, Buxbaum RE. Cytomechanics of axon development. Cell Biochem Biophys 1995;27(3):135-155.

34. Yu X, Bellamkonda RV. Tissue-engineered scaffolds are effective alternatives to autografts for bridging peripheral nerve gaps. Tissue Eng 2003;9:421-430.

35. Verdu E, Labrador RO, Rodriguez FJ, Ceballos D, Fores J, Navarro X. Alignment of collagen and laminin-containing gels improve nerve regeneration within silicone tubes. Restor Neurol Neurosci 2002;20:169-179. 